\title{
Les pratiques musicales chez les African Hebrew Israelites à l'épreuve de la mutation culturelle
}

Florian Mazzocut

\section{(2) OpenEdition}

Édition électronique

URL : http://journals.openedition.org/pa/99

DOI : 10.4000/pa.99

ISSN : 2273-0362

Éditeur

Université Lumière Lyon 2

Édition imprimée

Date de publication : 31 octobre 2012

Pagination : 50-78

ISBN : 1634-7706

ISSN : 1634-7706

Référence électronique

Florian Mazzocut, "Les pratiques musicales chez les African Hebrew Israelites à l'épreuve de la mutation culturelle », Parcours anthropologiques [En ligne], 8 | 2012, mis en ligne le 20 avril 2013, consulté le 20 avril 2019. URL : http://journals.openedition.org/pa/99 ; DOI : 10.4000/pa.99 


\section{Les pratiques musicales chez les African Hebrew Israelites à l'épreuve de la mutation culturelle}

\section{Florian Mazzocut}

Université Lumière Lyon 2, CREA

Les African Hebrew Israelites of Jerusalem sont une communauté afroaméricaine originaire de Chicago, qui s'est installée en Israël en 1969 sous la direction d'un leader charismatique reconnu comme le messie envoyé par Dieu pour conduire son peuple vers la liberté et la rédemption. Ils considèrent, à l'instar d'autres groupes religieux afro-américains issus du Black Judaism ${ }^{1}$ (Landing, 2002), qu'ils représentent les «vrais » israélites du texte biblique et s'efforcent, par une relecture de l'Ancien Testament, de reproduire le mode de vie des Hébreux par le biais de la réinvention d'un cadre culturel qu'ils appellent Divine Life Style. Ce dernier se définit comme un ensemble de règles et de pratiques qui couvrent tous les aspects de la vie individuelle et collective. Il est en grande partie l'œuvre du leader de la communauté, Ben Carter, plus connu aujourd'hui sous le nom de Ben Ammi Ben Israël. Aujourd'hui, les African Hebrew Israelites se considèrent comme une véritable « petite nation », partagée entre Israël et les Etats-Unis, dont le Divine Life Style et la reconnaissance de Ben Ammi comme messie et leader constituent le principal ciment identitaire.

Pour des auteurs comme Hans Baer et Merrill Singer (Baer \& Singer, 1992), les African Hebrew Israelites représentent une des expressions les plus radicales $\mathrm{du}$ nationalisme noir, dont l'action politique s'exprime dans le cadre de la pratique religieuse. Ils appartiennent à la tendance «messianiquenationaliste » de ce que James E. Landing (2002) définit comme le courant du Black Hebrewism, c'est-à-dire qu'ils redéfinissent le concept de rédemption du peuple noir à la fois en dehors des cadres du christianisme (et particulièrement $\mathrm{du}$ mouvement baptiste) à partir duquel toutes les formes syncrétiques de judaïsme noir ont émergé aux Etats-Unis, et en dehors du cadre $\mathrm{du}$ judaïsme orthodoxe $q \mathrm{u}^{\prime}$ ils considèrent comme une imposture visant à écarter les Noirs du cœur du récit biblique². Ils n'acceptent pas l'idée de salut dans sa compréhension spirituelle, comme une promesse de vie après la mort,

${ }^{1}$ Pour une définition et une approche historique très complète du Black Judaism aux Etats-Unis d'Amérique, voir James E. Landing (2002).

2 Pour une perspective historique des relations entre Juifs et Noirs aux Etats-Unis d'Amérique, voir Nicole Lapierre (2012). 
mais définissent ce dernier comme la «capacité à comprendre et à appliquer les lois de Dieu » (Ben Ammi Ben Israël, 1982), qui s'appliquent sur Terre, ici et maintenant. En fait, l'idée même de "mort physique » n'est pas acceptée chez les African Hebrew Israelites: elle est associée à une idée de dégénérescence spirituelle qui se manifeste par l'abandon du corps par le «souffle divin » causé par la non application des lois de Dieu³. La rédemption du peuple noir passe par la prise de conscience de son origine divine et la mise en pratique de ce statut de "peuple élu » par le biais du Divine Life Style. Elle se manifestera d'un point de vue pratique par une immortalité physique des African Hebrew Israelites revenu à l'état d'innocence de la Genèse, celui d'Adam et Eve avant leur chute et leur expulsion du paradis terrestre 4 .

L'objectif des African Hebrew Israelites, qu'ils estiment aujourd'hui avoir en grande partie atteint, consiste à récréer sur Terre, au cœur d'Israël, les " conditions du paradis », un reflet de la perfection divine par le biais de leur édifice communautaire. En cela, leur projet s'apparente à l'idéal monastique définit par Isabelle Jonveaux (2011) à cela près que cet idéal de sanctification de la vie quotidienne chez les African Hebrew Israelites s'exprime au travers d'une communauté composée de familles qui se considèrent comme « divines ». Pour les African Hebrew Israelites, il ne s'agit pas d'un renoncement au monde, mais d'un renoncement à " un » monde, celui du mal, et la mise en place d'un nouveau monde délimité par les frontières de la communauté5. Les African Hebrew Israelites considèrent être gouvernés par les lois de Dieu et lui consacrent leur édifice communautaire dont Ben Ammi représente la figure politique majeure. Ils appellent cet ordre politique, qui se déploie en parallèle des lois de la société israélienne, et parfois même contre celles-cí, le Kingdom of Yah, en référence au Royaume de Dieu annoncé par la prophétie de Daniel (Daniel, 2 : 44).

Les premiers membres de la communauté ont quitté les Etats-Unis en 1967 pour aller dans un premier temps s'établir au Libéria. L'Afrique de l'Ouest représente dans leurs discours la terre où le grand exil des israélites a commencé par le biais de la traite négrière. Pour eux, l'épisode de l'exil des Hébreux en Egypte ne représente qu'une illustration du châtiment divin qui s'est répété par le biais de l'esclavage, et est appelé à se renouveler indéfiniment jusqu'à ce que les vrais israélites dédient complètement leurs vies à la recherche de la rédemption divine.

${ }^{3}$ A ce sujet voir John. L. Jackson Jr. (2009).

4 Notons que des croyances très proches se retrouvent dans d'autres mouvements nationalistes afro-américains, en particulier la Nation of Islam.

${ }^{5}$ Paul A. Hare (1998), anthropologue israélien, dans sa monographie de la communauté la décrira comme une tentative de création d'une réalité sociale "vertueuse ", basée sur un ensemble de «nouvelles traditions » (pagination?).

6 Par exemple, les règles du Divine Life Style relatives au mariage dans la communauté autorisent (et même encouragent) la polygamie, en dépit de son caractère illégal en Israël. 
Selon l'idée de Ben Ammi, dans sa quête pour sa propre rédemption, le peuple israélite se devait de repasser par les différentes étapes de son exil avant de prétendre à un retour vers la Terre promise, Israël. Après une expérience de vie de deux ans et demi au Libéria où les quelques centaines de fidèles de Ben Ammi se réduisirent à une petite poignée par la rudesse de la vie qu'ils y menèrent, les African Hebrew Israelites se tournèrent finalement vers Israël7. Ils y émigrèrent illégalement par petits groupes en se regroupant dans les villes du sud du pays, en plein désert du Néguev, non sans avoir tenté d'obtenir la nationalité israélienne au nom de « la loi du Retour ».

Malgré le refus du ministère de l'Intérieur, les African Hebrew Israelites s'établirent dans la ville de Dimona, où ils vécurent durant plus de vingt ans sous la menace d'expulsions, subissant de fréquentes arrestations de la part des autorités et dans des conditions socio-économiques extrêmement précaires $^{8}$. Durant toute cette période, pourtant, les African Hebrew Israelites regroupés au sein d'un ancien centre d'absorption ${ }^{9}$ devenu pour eux le "village de la paix » (Village of Peace ou Kfar Hashalom en hébreu) n'ont eu de cesse de parcourir le pays pour expliquer et diffuser leur "message » notamment par le biais de la musique.

Aujourd'hui, malgré ses quarante ans de vie en Israël, la communauté des African Hebrew Israelites est toujours dans une sorte $\mathrm{d}^{\prime}$ « entre deux » juridique : la majeure partie de ses membres ne dispose que de visas temporaires ou permanents, très peu possèdent la citoyenneté israélienne. Dans la recherche $\mathrm{d}^{\prime}$ une reconnaissance par l'Etat d'Israël et l'obtention d'une citoyenneté juridique, la communauté exprime son engagement envers Israël par une « citoyenneté de l'âme » (Markowitz, 2003), sorte de discours d'appartenance alternatif qui souligne le droit des individus et des groupes à s'affirmer tout en faisant correspondre les identités qu'ils ont eux-mêmes élaborées au sein d'états existants. Cette "citoyenneté de l'âme» se manifestera par de nombreuses initiatives de la part des African Hebrew Israelites pour se rapprocher de la société israélienne et en particulier des institutions nationales comme la Défense Nationale.

7 Pour une approche historique détaillée de l'arrivée des African Hebrew Israelites en Israël, voir James Landing (2002) et Yvonne Chireau \& Nathaniel Deutsch (2000).

${ }^{8}$ Le quotidien israélien Haaretz, dans sa version anglophone, conserve des archives assez complètes concernant cette communauté. Un épisode particulièrement marquant pour la communauté $y$ est relaté dans un article accessible en ligne : http:/ / www.haaretz.com/weekend/anglo-file/recalling-their-show-of-strength-1.357513

${ }_{9}$ Un centre d'absorption désigne en Israël un lieu de vie transitoire, généralement sous la forme d'un village composé de maisons pouvant loger une famille, et doté d'équipements sociaux et sanitaires collectifs. Les centres d'absorption sont apparentés à des camps de transit où les nouveaux immigrants sont logés en attendant que leurs dossiers soient traités par l'administration et que des aides sociales à l'insertion professionnelle leurs soient attribuées. Un centre d'absorption n'est pas un lieu fermé, les nouveaux migrants sont, en principe, libres de circuler dans le pays. 
Pourtant, c'est surtout au travers de ses productions musicales que la communauté est aujourd'hui reconnue en Israël : des musiciens African Hebrew Israelites évoluent au sein de nombreuses formations musicales israéliennes, certains de leurs groupes comme les Soul Messengers ou le New World Fire Choir sont régulièrement sollicités pour des prestations variant de l'animation de mariages à l'accompagnement musical d'émissions de télévision, et d'autres vont jusqu'à représenter Israël dans l'émission de télévision «urovision $» 10$.

L'intérêt du public israélien pour les productions musicales des African Hebrew Israelites vient surtout du fait que celui-ci les rattache à un univers plus large de la musique noire afro-américaine : en effet, la communauté a très tôt compris et exploité ce créneau en proposant des orchestres de reprises de " classiques » soul, jazz et reggae. Cependant, les pratiques musicales et les musiciens de Kfar Hashalom évoluent au sein du cadre culturel du Divine Life Style où la musique est considérée comme un élément central, rattaché à la quête de la rédemption et à la construction d'une identité nationale. Dans ce cadre bien particulier, le rôle de musicien se dédouble d'un rôle politique et religieux assimilé par l'idéologie de Ben Ammi à une fonction de prophète. Les pratiques musicales obéissent à un ensemble de principes normatifs très stricts et particuliers qui sont confrontés aujourd'hui à toute une série de mutations culturelles et musicales qui émergent des jeunes générations de la communauté. Ces mutations, dont nous étudierons une des expressions au travers de l'émergence de la musique rap, opèrent une remise en question inédite du cadre du Divine Life Style dans le contexte de vie contemporain de la communauté en Israël.

Dans un premier temps, nous présenterons la pensée du leader charismatique des African Hebrew Israelites en insistant sur certains aspects de son paradigme culturel, et la place que la musique y occupe. Nous articulerons ensuite cette pensée aux pratiques musicales des African Hebrew Israelites dans une perspective historique en montrant comment leurs musiciens sont perçus comme des modèles de réussite, combinant des aspects éthiques et économiques. Dans la dernière partie, nous verrons comment de jeunes artistes s'approprient la musique rap pour éclater et redéfinir le cadre conceptuel du Divine Life Style, afin de négocier au sein de la communauté leur place de musiciens-prophète

10 En 2006, c'est le chanteur Eddie Butler, membre de la communauté des African Hebrew Israelites, qui a représenté Israël à l'Eurovision avec le titre Together we are One. 


\section{LA MUSIQUE COMME ACTIVITÉ VERTUEUSE : LA FIGURE DU MUSICIEN-PROPHÈTE}

\section{La musique, un élément culturel du Divine Life Style}

Au cours de leur histoire, les African Hebrew Israelites se sont à plusieurs reprises interrogés sur la signification à accorder à leur culture et à leur appartenance culturelle. Pour eux, la culture est perçue comme «le reflet des éléments fondamentaux qui se combinent pour constituer une société (...) chaque élément s'y inscrit comme un facteur signifiant car il est une partie du tout qui compose une personnalité individuelle ou une partie des normes d'une société (...) Quand nous décrivons chaque élément de notre environnement social comme "divin", cela couvre tous les champs sur lesquels la souveraineté de Yah se déploie dans nos vies et nos expressions »11.

La culture est donc une chaîne d'éléments qualifiée $d^{\prime}$ " holistique », répondant, dans une conception assez fonctionnaliste, à divers niveaux de besoins. Chacun de ces "éléments » doit en outre être clairement identifiable comme relevant de la culture du Kingdom of Yah, c' est-à-dire être produit dans le cadre de la communauté : "La culture divine est la marque qui assure l'identité d'un peuple droit et est essentielle pour assurer l'existence de l'éternel, Yah vivant au milieu de son peuple (...) La culture est le baromètre des valeurs morales d'une société et peut même déterminer l'ampleur et la longévité d'une suprématie » (Taahmenyah bath Shaleak et Ahmadiel Ben Yehuda, date d'édition non précisée).

Dans son ouvrage, God, the Black Man and Truth (1982), Ben Ammi interroge une à une les pratiques culturelles des Afro-Américains ${ }^{12}$, en expliquant à quel point la société occidentale peut dénaturer une culture divine jusqu'à en faire oublier sa signification à ses représentants. En analysant et en critiquant ce qu'il qualifie de "pratiques culturelles d'esclaves" dans l'Amérique des années 1970, Ben Ammi propose de construire un nouvel ordre culturel basé sur une réinterprétation de l'héritage socio-culturel afro-américain lu au prisme de l'histoire des Hébreux. Cet héritage, selon le leader, ne doit plus être compris dans une posture conciliante et intégrée à une société «blanche ", dominante et oppressante: il s'agit d'un héritage divin mis en danger et perverti par un environnement corrompu dont il convient de se détacher de la façon la plus radicale possible : la séparation pure et simple.

Ainsi, de la façon de s'habiller, de se nourrir jusqu'aux modalités de fondation d'une famille, Ben Ammi, en s'appuyant sur la Bible, s'efforce de faire prendre conscience de l'identité «israélite» du peuple noir. Or, la musique se trouve êtres abordée dès le premier chapitre de son premier

${ }^{11}$ Cette définition de la culture est extraite de l'introduction du code vestimentaire des African Hebrew Israelites, édité par le Ministère de l'Information de Kfar Hashalom et ses représentants, Taahmenyah bath Shaleak et Ahmadiel Ben Yehuda (date d'édition non précisée).

12 Ben Ammi «délimite» le peuple israélite aux descendants d'esclaves exilés en Amérique du Nord et dans les Caraïbes. 
ouvrage. Ce chapitre est intitulé «La quête de Dieu » et le passage concernant la musique est répertorié sous le titre de « La puissance de la musique et de la danse » :

«La musique est bien plus qu'une forme de divertissement. La musique identifie les races, les nationalités et les communautés. Elle a des effets hypnotiques de grande envergure sur le cerveau et l'âme et peut prendre complètement le contrôle du corps et de l'esprit. La musique peut déterminer l'humeur d'une personne ou d'un peuple. Après avoir compris ses profonds effets sur l'esprit, il devient évident que nous devons y porter notre attention car ces effets peuvent être bons ou mauvais. La musique est comme une série de vagues de pensée qui poussent les hommes à penser et à faire le bien ou le mal, de bonnes choses ou des choses stupides. En plus de cela, il y a des sons qui détruisent les tympans, brisent des ampoules et font craquer le verre. Nous devons aussi nous demander : existe-t-il une forme de son qui peut détruire l'esprit? La réponse est certainement oui. Par ailleurs, il y a l'esprit du musicien dont les vagues de pensées sont transmises au travers de sa musique. Examinons ce point plus minutieusement en prenant la musique "populaire, club et rock" comme un exemple ${ }^{13}$... » (Ben Ammi Ben Israël, $1982: 27$; traduction personnelle)

L'importance du fait musical dans la (re)construction d'un édifice culturel se manifeste d'abord pour Ben Ammi d'un point de vue politique : la musique est perçue comme une véritable énergie, une source $d$ 'inspiration qui dirige l'homme dans sa vie en suscitant des "images ", des vagues de pensées. En cela, il est intéressant de noter que le leader ne conçoit absolument pas la possibilité de se passer d'une activité musicale. Il la considère au contraire comme un élément culturel crucial en dépit d'une nature ambivalente du fait musical. Selon lui, cette nature ambivalente est intimement liée aux modalités de production de la musique, c'est-à-dire à son rapport avec le musicien. Lors de l'expérience musicale, l'esprit du musicien entre en communion avec celui du public et cette expérience se vit comme un rapport de force où c'est de l'esprit de ce musicien que va dépendre la teneur morale des «vagues de pensées » qui vont être perçues.

13 "Music is much more than a form of entertainment; music identifies races, nationalities and communities. It has far-reaching, hypnotic effects on the brain and soul, and can take complete control of the body and mind. Music can determinate the mood of a person or a people. After comprehending its profound effects on the mind, it stands to reason that we must beware, for the effects can be good or evil. Music is like a series of thought waves that cause men to think and do right or wrong, wise things or foolish things. In addition to that, there are sounds that destroy ear drums, shatter light bulbs, and crack glass. We must also ask ourselves: is there a mode or musical sound that can destroy the mind? The answer is certainly yes. Furthermore, there is the mind of the musician whose thoughts are transmitted through his music. Let us examine this ;point more thoroughly using "popular, club and rock" music as an example..." (Ben Ammi Ben Israël, 1982: 27) 


\section{Les Songs of Delivrance comme cadre normatif des pratiques musicales}

Il semble ici qu' un choix soit sous-entendu par le leader : le choix d'accepter d'écouter ou de produire une musique potentiellement "néfaste » avec des musiciens conditionnés par des mentalités « d'esclaves » ou au contraire une musique " positive », avec des musiciens dont on est absolument certain de la valeur morale. Pour s'assurer de ce caractère positif, Ben Ammi propose de procéder à un examen des références à la musique dans la Bible et des activités musicales qui y sont mentionnées. Il utilise principalement des versets de l'épître de Jean (Jean 1:1) et du livre des Nombres (Nombres $10: 8-9$ et $31: 6)^{14}$ : dans son travail d'interprétation, le concept de «parole » y est immédiatement assimilé à celui de « son », puis de « musique».

«Il y avait un son avec Dieu au commencement; toutes les choses furent faites par le son, et sans le son rien n'aurait pu avoir été fait. A partir de cela nous savons qu'il y a une puissance créatrice dans le son, car dans la Genèse un son bénéfique a amené la vie. Pour que les gens comprennent l'importance du son, Dieu a appris à nos prédécesseurs à faire retentir les trompettes d'une certaine forme de musique pour le rassemblement des masses. Il y avait aussi une musique spéciale qui était jouée quand venait le temps de partir en guerre. Dieu a permis le succès entier sur les forces du mal autour d'un son, comme IL commanda nos pères de jouer un certain son s'ils étaient sous l'oppression de leurs ennemis. Quand ce son était entendu, Il entendait, se renforçait et sauvait. Est-ce que Dieu ne tiendrait pas Sa parole? Est-ce que nos musiciens ont considéré la vraie spiritualité de la musique ? Se sont-ils tous égarés? N'y en a-t-il aucun qui comprenne? Ne devraiton pas au moins appliquer cette ancienne sagesse dans notre lutte pour la liberté ? Il y a ceux qui ont des intentions maléfiques qui sont constamment en train de chercher et de manipuler les contenus des écritures et qui utilisent ces mêmes instructions contre nous. Référons-nous à l'histoire de la cavalerie américaine dans leur guerre contre les indiens d'Amérique »15 (Ben Ammi Ben Israël, 1982 : 28-29 ; traduction personnelle).

14 « Au commencement était la Parole, et la Parole était avec Dieu, et la Parole était Dieu » (Jean $1: 1)$

15 "There was a sound with God in the beginning; all things were made by sound, and without sound nothing was made. From this we know there is a creative power in sound, for in Genesis a right sound brought forth life. In order that the people know the importance of sound, God instructed our predecessors to make trumpets to play a certain mode of music for the assembling of the masses. There was also a special music played when it was time to go to war. God evolved the entire success over evil forces around a sound, as He commanded our fathers to play a certain sound if they were under the oppression by their enemies. When this sound was heard, He would hear, strenghten and save. Will God not keep His word ? Have our musicians considered the true spirituality of music? Have they all gone astray? Are there none that understand? Should we not at least apply this ancient widsom in our struggle for freedom? There are those of evil intentions that are constantly searching the scriptures and manipulating the contents and using these same instructions against us. Let us reflect on the history of the U.S. Cavalry in their war against the American Indians" (Ben Ammi Ben Israël, 1982 : 28-29). 
$\mathrm{Au}$ travers de ce passage Ben Ammi propose une articulation de l'activité musicale avec la recherche de la véritable identité des Afro-américains. Il va même plus loin en esquissant un des principaux champs d'application de l'activité musicale: la guerre et la lutte contre l'oppression via le pouvoir fédérateur et identitaire de la musique.

Ben Ammi suggère que la véritable force de la musique, et particulièrement de la musique noire, a été vidée de sa substance et même pire, qu'elle s'est faite dérober ses marqueurs les plus sacrés par «ceux qui ont des intentions maléfiques ». Ces derniers ne se contentent pas de pervertir les attributs du peuple de Dieu, mais sont également les agents d'un vaste projet démoniaque visant à faire oublier aux descendants des Hébreux leur vraie identité en «brouillant les pistes » ${ }^{16}$.

Pour Ben Ammi, c'est la création de catégories musicales actuelles dites « mixtes » ou de «variétés » (crossover) qui génère le plus de confusion. Il assimile les artistes noirs qui s'inscrivent dans ces catégories à des artistes qui ont « vendu l'âme de la musique noire » (Ben Ammi, 1982 : 30).

Il est donc du devoir de l'artiste noir de comprendre la dimension divine de son travail. Pour ce faire, Ben Ammi va procéder à un travail de mise en perspective historique de ce qu'il considère comme relevant de la musique noire, héritière d'une culture qui, comme le souligne le ministre de l'Information de Kfar Hashalom lors d'une interview par la chaîne Russia Today en Avril 2012 : "Ne chante pas à propos du Mali ou du Ghana, mais qui chante Canaan, Jérusalem et Jéricho ».

« Nous devons corriger la terminologie moderne utilisée pour décrire nos formes de musique, car à plusieurs niveaux les termes sont très déroutants. Il est impératif que les noms originaux soient compris et rappelés. Le nom original de ce que nous appelons le Blues était la musique des lamentations. Le nom original pour la musique d'église était le Gospel ou les Chants de Sion. Le nom original de ce que l'on appelle maintenant le Jazz ou le Modern Jazz- vous serez peut être choqué de l'apprendre - étaient les Spirituals ou Spiritual. La musique Soul a conservé son nom original, mais a complètement été détournée son contenu. La musique impie est basée sur la création de doute, généralise une insouciance gratuite, le désir sexuel, et fait apparaître la perversité comme une chose plaisante (Sexual Healing, Part-time Lover, Thigh Ride). Nos autrefois pures, créatives expressions musicales pleines de pouvoir de guérison et de force spirituelle - sont devenues frelatées, ont été mises dans un état d'impiété, et sont donc, dans leur forme présente, inutiles dans le combat pour la droiture (la quête de Dieu). (...) Est ce qu'en substance tous les chants de Gospel ne sont pas à propos de Jéricho, Jérusalem, la Galilée, le

${ }^{16}$ Là aussi la parenté des croyances prônée par les African Hebrews Israelites avec celles de la Nation of Islam est évidente. A l'instar des African Hebrew Israelites, ce mouvement religieux afro-américain déploie une rhétorique identitaire au sein de laquelle l'homme noir doit se réapproprier son identité musulmane originelle. Comme le souligne Pauline Guedj (2003), on assiste également au sein de la Nation of Islam à une vive condamnation de l'église et du christianisme comme les agents de la confusion et de la destruction de l'identité noire. 
Jourdain et notre Terre ISRAËL ?! Le Gospel dans sa forme pure était notre appel collectif pour la compréhension, la pitié et la clémence. C'était pour garder nos cœurs et nos souvenirs vers notre Terre, Israël (... Si j'oublie ton nom, ô Jérusalem...) et pour garder vivant notre espoir d'un jour y retourner ${ }^{17}$ (Ben Ammi Ben Israël, 1982 : 30-32 ; traduction personnelle).

Ben Ammi déduit ici une généalogie directe des musiques du Temple avec des styles de musique afro-américains. Selon lui, ces formes musicales autrefois de nature divine ont été réduites à des formes impies, venant grossir les rangs, en quelque sorte, de l'arsenal musical des forces maléfiques qui dirigent le monde et maintiennent le peuple de Dieu en esclavage.

Ce qu'il propose, loin d'abandonner ces musiques, c'est de les faire entrer dans un processus de "rédemption ", le même que celui par lequel doivent passer l'homme et la femme noirs. Pour cela, il est nécessaire d'opérer une relecture critique de ces formes musicales pour les rétablir dans leurs formes originales et comprendre leur place centrale dans la « nouvelle vision » de la culture que le leader propose. Ben Ammi opère ce faisant une dissociation nette entre la «forme » et le « fond » $d^{\prime}$ une production musicale.

La forme de la musique est constituée par sa dimension rythmique et mélodique, elle a une profonde incidence psychologique sur les auditeurs. Ainsi, Ben Ammi rejette d'emblée certaines formes musicales comme le rock n'roll qu'il accuse de généraliser la débauche par des rythmes décadents. Pour ce qui est de la musique noire, le leader adopte un point de vue différent : les formes musicales comme le jazz, la soul ou le blues sont "correctes » d'un point de vue musical. Et c'est d'un point de vue du «contenu » idéologique qu'il s'agit de les réformer.

Ainsi, la « réforme » de la musique noire qu'il propose se concentre d'abord sur les paroles et les textes, c'est-à-dire sur les images véhiculées par la musique, plus que sur les formes musicales elles-mêmes qui vont rester très proches des styles musicaux afro-américains comme la soul, le gospel ou le

17 "We must correct the modern terminologies used to describe our forms of music, for many instances the terms are very misleading. It is imperative that the original names be understood and revived. The original name for what we call the Blues was the music of Lamentations. The original name for church music was Gospels or the Songs of Zion. The original name for what is called Jazz or Modern Jazz- you may be shocked to know- was Spirituals or the Spiritual. Soul music has held its original name but is completely foreign in its content. Music of unrighteousness is based upon creating mistrust, arousing wanton, sexual desire, and making wickedness appear pleasant (Sexual Healing, Part-time Lover, Thigh Right). Our once pure, creative musical expressions - full of healing power and spiritual strength - have become adulterated, been made ungodly and are therefore, in their present form, useless in the struggle for righteousness (quest for God). (...) Are not virtually all Gospel songs about Jericho, Jerusalem, Galilee, the Jordan, our Land ISRAEL? The Gospel in its pure form was our collective plea for undestanding, mercy and forgiveness. It was to keep our hearts and remembrance on our Land Israel (...if I forget thee o Jerusalem...) and to keep alive our hope to someday return there" (Ben Ammi Ben Israël, 1982 : 30-32). 
reggae. Tous ces styles musicaux seront regroupés sous une seule appellation, "les Chants de la Délivrance » ou Songs of Delivrance définis comme : "Une toute nouvelle et existante catégorie de son - un genre musical unique et édifiant offrant l'espoir, la motivation et l'inspiration divine à ses auditeurs, aussi bien qu'une compréhension pratique, non mythique, du chemin qui mène à la vraie délivrance. Ces chants sont enracinés dans l'accessible et la vérifiable vérité de Yah - Le Créateur et son univers. Les mélodies, bien que de formes variées, proviennent toutes de Jérusalem, soulignant et promulguant les attentes terrestres vertueuses (la vie) promises par le Créateur, ici et maintenant.» 18

De plus, les Song of Delivrance expriment au travers de leurs paroles un univers symbolique et national qui les rapproche de certains styles musicaux, comme le reggae, rattaché au mouvement rastafari. Martina Könighofer (2008 : 87) soulignera d'ailleurs un certain nombre de correspondances entre la doctrine des African Hebrew Israelites et les «Sept principes du rastafarisme » détaillés par Winston William (2000 : 16-22).

Le reggae de Bob Marley est considéré comme une référence musicale à Kfar Hashalom. Les formations musicales de la communauté jouent également un certain nombre de reprises des Wailers ("No woman no cry", "Redemption song ", etc.). Pourtant, ces morceaux font l'objet de nombreux commentaires et critiques malgré leur mobilisation de symboles communs avec les Songs of Delivrance des African Hebrew Israelites. Ces derniers considèrent qu'une certaine incompréhension de la nature divine du peuple noir a conduit Bob Marley à relayer certaines erreurs dans ses textes, comme le fait que l'Ethiopie y soit considérée comme la Terre promise, et l'Afrique n'y soit pas associée directement avec la terre d'Israël. Le décalage généré par cette interprétation $\mathrm{du}$ texte biblique couplé au fait que les rastafaris ne considèrent pas Ben Ammi comme le messie font que les principes du rastafarisme, même relayés par une musique aussi appréciée que celle de Bob Marley au sein de Kfar Hahsalom ne rentre pas exactement dans le cadre des Songs of Delivrance.

La musique est donc pensée dans un cadre spécifique des Songs of Delivrance, qui renvoie à la fois à des styles musicaux considérés comme noirs, à un contenu symbolique en adéquation avec l'idée de rédemption de Ben Ammi, et, surtout, à des modalités de création engageant les musiciens de la communauté. Ces derniers, nous allons le voir, sont au même titre que leurs créations, appelés à reconsidérer leur rôle au prisme de la figure du musicienprophète inspiré par la Bible.

\section{La figure du musicien-prophète}

Dans God, the Black Man and Truth, Ben Ammi (1982: 34) esquisse les contours d'un «modèle musical » et par extension d'un "musicien modèle ». Ce dernier doit être doté, en plus des compétences propres à la pratique

\footnotetext{
18 Plaquette de présentation du label Songs of Delivrance (2009).
} 
musicale (technicité, enthousiasme, ingéniosité, etc.), de valeurs morales irréprochables. Il est pleinement conscient de la signification profonde et «vertueuse » de sa musique et devient, dans sa façon d'être et de jouer, un prophète :

"Cette compagnie de prophètes ${ }^{19}$ dotés d'instruments de musique était un groupe de musiciens. La mission d'un prophète était de répandre la Parole de Dieu. Ainsi, ces prophètes, musiciens, jouaient la Parole de Dieu, le son de Dieu aux hommes. Les musiciens doivent comprendre qu'il y a une corrélation directe entre le son et l'esprit Noir. En étant dirigés par la Parole de Dieu, nous voyons que Dieu a utilisé la musique pour gouverner et protéger nos pères. L'histoire en appelle maintenant au prophète-musicien pour s'avancer et une fois encore « jouer la Parole de Dieu ». Je sais que vous vous rappelez que dans le folklore Noir, la fin de la captivité était signalée par l'ange Gabriel "sonnant son cor». Celui qui a des oreilles pour entendre, laissez le entendre. Gabriel fais sonner ton cor, fais sonner ton cor, fais sonner ton cor ! »20 (Ben Ammi Ben Israël, $1982: 34$; traduction personnelle).

Le musicien modèle, ce musicien-prophète est celui chez qui l'on retrouve ces qualités éthiques, et dont tous les aspects de la personnalité sont tournés vers l'idée de rédemption transmise par la musique. En d'autres termes, ce musicien modèle ne peut finalement se rencontrer que dans un contexte qui fait de la quête de Dieu une priorité absolue, ainsi que le propose le schéma de transformation culturelle radicale de Ben Ammi. Il est donc avant tout un musicien communautaire des African Hebrew Israelites of Jerusalem qui a compris et intégré ces valeurs.

Le musicien est, du point de vue du leader, un enjeu de toute première importance : sous réserve qu'il ait assimilé le caractère divin et prophétique de sa fonction, il participera, par sa capacité à «jouer la Parole de Dieu » à renforcer le « peuple de Dieu » dans sa lutte pour la liberté.

En effet, la pratique musicale au sein de la communauté a toujours accompagné les événements importants et est, encore aujourd'hui, un élément central dans les célébrations religieuses (comme le service de Shabbat) et

${ }^{19}$ Ben Ammi se réfère au premier livre de Samuel : " Après cela tu viendras à Guibéa de Dieu, où sont les colonnes des Philistins, et là, en entrant dans la ville, tu rencontreras une troupe de prophètes descendant du haut-lieu, précédés de luths, tambourins, flûtes et harpes, et prophétisant. Et l'Esprit de l'Eternel te saisira et tu prophétiseras avec eux et tu seras changé en un autre homme » ( I Samuel $10: 5-6$ ).

20 "This company of prophets with musical instruments was a band of musicians. The mission of a prophet was to speak the word of God. Thus these prophets, musicians, played the Word of God, the sound of God unto the people. The musicians must understand that there is a direct correlation between sound and the Black mind. Being led by the Word of God, we see that God used musical sound in governing and protecting our fathers. History is now calling for the prophet-musician to come fort hand once again " play the Word of God ». I know you recall that in the Black folklore, the end of captivity was signaled by Gabriel the angel «blowing his horn ». He that has ears to hear, let him hear. Gabriel! Blow your horn, blow your horn, blow your horn!" (Ben Ammi Ben Israël, 1982 : 34). 
nationales (Memorial Passover ou New World Passover). Selon des modalités différentes, les musiciens de la communauté se produisent en solo, en chœurs ou en orchestres complets pour accompagner les paroles des prêcheurs, des leaders ou des conférenciers. La musique est véritablement un outil de mise en scène du pouvoir et également du savoir. Toutes les conférences et réunions sont accompagnées de musiques ou de chants, en ouverture, en intermèdes ou en fermeture.

De même, lorsque le leader Ben Ammi Ben Israël se déplace de sa maison au sein du Kfar HaShalom jusqu'aux salles communautaires pour y délivrer des enseignements ou des messages, il est accompagné à la fois par une escorte de "garde du corps » rompus aux techniques de combat, mais aussi par des prêtres qui dirigent un orchestre de percussions ainsi qu'un chœur complet vêtu de blanc. Ces chœurs rythment les pas du leader et annoncent son arrivée imminente au public qui se lève et fait silence.

Dans le cadre des événements collectifs, toutes les générations sont représentées et proposent des performances variées. Mais qu'il s'agisse de performances musicales acoustiques, amplifiées électroniquement, "live » ou enregistrées et distribuées sur CD ou DVD, toutes se doivent d'obéir aux normes mises en place par le leadership communautaire. Ces normes, qui font se rejoindre politique et esthétique, sont sanctionnées par la mise en place, dès les années 1980, d'un label "Songs of Delivrance ». Ce label se trouve être à la fois pensé comme un symbole identitaire fort en relation avec l'idéologie de Ben Ammi Ben Israël (l'idée est de ne plus faire de gospel, de blues ou de soul, mais de produire une musique rédemptrice, consciente et divine: les "musiques du Temple ») et à la fois comme un label doté d'une logique commerciale permettant d'identifier et de rattacher une création musicale à un certain contexte de production et d'édition (les affaires communautaires et leurs standards de production).

Mais comment les formations de musiciens des African Hebrew Israelites of Jerusalem articulent-ils cette idée de rédemption par la musique avec les réalités concrètes de leur vie en Israël ? Nous allons maintenant interroger les modalités de pratique de la musique dans la communauté, ainsi que la place qu'elle y a peu à peu occupée dans le dispositif éducatif des African Hebrew Israelites.

LES PRATIQUES MUSICALES À KFAR HASHALOM ENTRE RESSOURCES ÉCONOMIQUES ET PLATEFORME PÉDAGOGIQUE

\section{Le rôle des musiciens communautaires dans l'établissement du Kingdom of Yah en Israël}

Pour les adultes vivant à Kfar Hashalom, l'arrivée en Israël renvoie à des souvenirs de nature très ambivalente. L'enthousiasme suscité par la « révélation » de Ben Ammi et par la fin des épreuves du Libéria, s'est couplé 
à tout un ensemble de nouveaux problèmes relatifs à l'installation sur la «Terre promise». Comme expliqué précédemment, le refus des autorités israéliennes de leur accorder la citoyenneté, en dépit de leurs exigences ${ }^{21}$, s'est soldé par une longue période de tensions entre ces dernières et les représentants de la communauté.

D'un point de vue social et historique, les African Hebrew Israelites soulignent deux tensions majeures qui caractérisaient leur vie à cette époque : une sensation d'oppression et de rejet de la part des autorités israéliennes et, par extension, des populations locales dans les villes du Néguev, et un sentiment d'insécurité et de dislocation au sein même de l'espace communautaire.

Le premier phénomène, que certains African Hebrew Israelites expliquent vivre encore aujourd'hui, a eu pour principal corollaire une situation juridique et économique précaire, limitant drastiquement l'accès aux emplois légaux, ainsi qu'aux possibilités de développement et d'investissement foncier.

Le deuxième phénomène, quant à lui, est encore aujourd'hui sujet à un certain tabou, chez les Israéliens comme chez les African Hebrew Israelites ${ }^{22}$. Il a contribué d'une part à générer des épisodes de tensions internes où la légitimité de Ben Ammi Ben Israël en tant que leader a été fortement contestée, et d'autre part a eu pour effet d'inciter des fidèles découragés à s'intégrer à la société israélienne en procédant, au besoin, à une conversion au judaïsme, pourtant rejetée avec ardeur par le leadership communautaire.

$\mathrm{Au}$ sein de ce contexte particulier, les formations musicales des African Hebrew Israelites of Jerusalem, et particulièrement des groupes comme les Soul Messengers ${ }^{23}$, ont joué aux niveaux économique et politique un rôle central dans le maintien de la communauté en Israël.

Ce rôle central s'est d'abord manifesté sur le plan économique. La répartition des ressources à Kfar Hahsalom repose sur un modèle qui se veut assez proche du modèle du Kibboutz: l'idée de propriété privée se limite aux possessions matérielles personnelles (objets, vêtements, etc.), et les ressources

${ }^{21}$ Une ancienne employée du ministère de l'Intérieur, chargée de traduction, présente au moment de l'arrivée des premiers African Hebrew Israelites en Israël nous a confié son incrédulité et celle de ses collègues lorsqu'elle a du traduire leur demande aux autorités.

22 Cette situation, en plus d'avoir été vécue comme un épisode dégradant pour les African Hebrew Israelites, a été marquée par des épisodes de violence au sein de la communauté où au moins un membre, contestant le leadership communautaire de Ben Ammi Ben Israël, a trouvé la mort en pleine rue à Dimona, battu à mort par ses anciens camarades. Cet épisode tragique a profondément choqué les habitants de Dimona et a largement concouru à dégrader l'image de la communauté à un niveau local.

${ }^{23}$ Les Soul Messengers sont un des groupes communautaires les plus connus en Israël. A leur formation ils se composaient d'un orchestre de douze musiciens et choristes ainsi que d'un groupe de danseurs. Ils se sont illustrés dès les années 1970 en Israël comme un groupe de compositions et de reprises puisant dans un répertoire de musique soul, jazz et reggae, en adaptant des musiques comme celles des Temptations, de James Brown, de Harry Bellafonte et plus tard de Bob Marley. 
financières permettant aux fidèles et à leurs familles de vivre sont centralisées par un ministère de $l^{\prime}$ Economie et redistribuées à chaque famille selon ses «besoins ». En principe, les recettes permettant à ce système de fonctionner sont collectées sous forme de taxes, alimentées en grande partie par les infrastructures commerciales de la communauté (restaurants, boutique, etc.). Or, à leur arrivée en Israël, les African Hebrew Israelites, ne disposaient pas encore de ces infrastructures. Leurs principales sources de revenus locales étaient générées par les prestations musicales de groupes comme les Soul Messengers, se frayant peu à peu un chemin sur la scène israélienne, se produisant fréquemment dans des cérémonies de mariages, de bar-mitsva dans tous le pays.

Pendant des années donc, les formations musicales à Kfar Hahsalom ont eu un rôle pivot de captation financière qui leur a conféré un poids politique non négligeable à la fois au sein de la communauté et dans la société israélienne par la constitution de réseaux de clientèle plus ou moins stables.

Sur le plan politique, le rôle des formations musicales s'est manifesté principalement dans sa fonction de soutien au leader Ben Ammi Ben Israël. Ce soutien s'explique en grande partie par le fait que les premiers fidèles de Ben Ammi étaient eux-mêmes musiciens, et d'un point de vue hiérarchique, ils occupaient déjà des postes politiques au sein du leadership. Les musiciens constituaient véritablement le noyau idéologique de la communauté, prenant part aux décisions et aux nouvelles orientations décidées par le leader. Ainsi, en plus d'être les principales ressources économiques, les groupes comme les Soul Messengers ont été les premiers appuis de Ben Ammi dans ses efforts pour le maintien d'une certaine cohésion dans la communauté et l'application des règles du Divine Life Style.

À ce niveau, les musiciens-prophètes de Kfar Hahsalom se sont illustrés comme des auxiliaires de maintien de l'ordre politique, endossant au besoin de multiples casquettes de chantres de Ben Ammi, de bras droits et d'entrepreneurs au service de la communauté.

Les musiciens accompagnaient les prêches du leader, des prêtres, ainsi que certaines tâches de la vie quotidienne ce qui a certainement eu des effets fédérateurs quand les African Hebrew Israelites traversaient une période difficile. Toutefois, le soutien politique à Ben Ammi de la part des musiciens a aussi pris une teneur beaucoup plus directe en s'exprimant par des fonctions de médiations ou de coercitions au sein de la communauté, très éloignée de la pratique musicale elle-même.

Dans le contexte bien particulier des premières années d'implantation en Israël, ces diverses formations musicales, tout en revendiquant leur appartenance aux Songs of Delivrance, déployaient leurs activités dans un objectif de «survie » où la logique de concurrence commerciale n'était pas présente. On assistait ainsi à une rotation fréquente du personnel de ces groupes, leur permettant au besoin de se produire sans arrêt, dans une logique de non-concurrence. 
Les groupes communautaires aujourd'hui : entre logique commerciale et symboles nationaux institutionnalisés

Depuis les années 1990, la situation socio-économique de la communauté a changé. L'Etat d'Israël a reconnu, sous certaines conditions on l'a vu, la volonté des African Hebrew Israelites de rester durablement sur le territoire : l'accès aux visas a été facilité et des droits à certaines aides sociales (israéliennes mais aussi des Etats-unis) ont été ouverts. Les African Hebrew Israelites ont été regroupés dans l'ancien centre d'absorption de Dimona qui est devenu le Kfar Hashalom, leur centre spirituel. Les Etats-Unis, par le biais du ministère des Affaires étrangères, ont financé certains équipements communautaires (l'école et le gymnase), et plusieurs activités commerciales ont émergé, notamment dans le secteur de la restauration. Des restaurants et des boutiques appartenant à la communauté et gérés par des gestionnaires semi-indépendants ouvrent dans des villes israéliennes, dont plusieurs à TelAviv. Toutes ces nouvelles structures sont autant de sources de financement pour l'économie communautaire par le biais de taxes exprimées sous formes de «dons ». À cela s'ajoute un accès plus important, bien que toujours assez restreint, à des emplois légaux sur le marché du travail israélien. Ces changements ont eu pour principale conséquence une dépendance financière moindre d'une partie de la communauté à l'égard du leadership communautaire et de son ministère de l'Economie. Ce dernier a pu davantage concentrer ses actions de financement sur le développement de nouveaux secteurs d'activités et limite sa redistribution financière directe aux familles qui sont le plus dans le besoin. Chacun dispose désormais librement de ressources financières propres bien qu'inégales en proportions.

Cette transition vers une nouvelle donne socio-économique a également conduit à une mutation importante au sein des groupes musicaux communautaires. Ceux-ci, forts de leur expérience de près de quarante ans de prestations musicales en Israël, basent leurs activités sur des réseaux de clientèle stables et jouissent d'une reconnaissance locale et internationale.

$\mathrm{D}^{\prime}$ un point de vue économique, ces groupes inscrivent désormais leurs pratiques dans une logique de profit personnel et de carrière musicale. Ils s'apparentent davantage à de petites institutions qui «fonctionnent » de manière autonome, et dont les membres fondateurs ne font plus partie.

En témoignent un certain nombre d'indices visibles, comme le recrutement de musiciens israéliens extérieurs à la communauté dans la nouvelle formation des Soul Messengers, ou encore une inscription dans la durée au sein de certains secteurs comme l'hôtellerie des complexes touristiques de la Mer Morte au travers de contrats d'animation permanents. Il faut également mentionner la mise en place d'un studio d'enregistrement professionnel à Dimona, produisant les artistes de la communauté et possédant un label du nom de Songs of Delivrance. 
D'un point de vue politique, le rôle des formations musicales a également évolué. À leur reconnaissance par le public israélien, fait désormais écho une célébrité au sein même de la communauté, qui les acclame comme de véritables modèles de réussite. Les groupes comme les Soul Messengers ou le New World Fire Choir sont considérés comme de véritables «monuments musicaux », en relation avec l'histoire des African Hebrew Israelites en Israël. En marge de leurs activités professionnelles, ces groupes se produisent fréquemment à Kfar Hashalom pour des célébrations importantes, réintégrant pour l'occasion les anciens membres du groupe. Ces concerts commémoratifs accompagnent des cérémonies nationales ou personnelles (anniversaires, mariages, etc.) et sont l'occasion de rejouer les premiers succès des musiciens du Kingdom of Yah.

Ces musiciens appartiennent aujourd'hui encore pour la plupart au leadership communautaire ou du moins possèdent les titres les plus prestigieux dans la communauté (princes, ministres, frères ou sœurs couronnés). La reconnaissance de la réussite économique se double donc d'une forme de réussite politique, acquise par le biais de l'éducation aux jeunes générations de Kfar Hahsalom ${ }^{24}$. La figure du musicien-prophète formulée par Ben Ammi trouve donc un écho « réel et bien vivant » au travers des musiciens adultes et de l'institution de la musique comme une composante majeure de l'éducation dans la communauté.

\section{Musique et éducation à Kfar Hahsalom: la transmission du modèle du musicien-prophète}

Les jeunes générations de Kfar Hashalom sont dans un rapport constant avec la pratique musicale : celle-ci est enseignée comme une part de leur histoire, de leur culture - en tant qu'élément du Divine Life Style - et comme un éventuel secteur professionnel vecteur d'une réussite et d'une reconnaissance incarnées par de nombreux exemples dans leur environnement direct.

L'approche du fait musical dans le domaine de l'éducation à Kfar Hashalom comporte un volet théorique et un aspect pratique. C'est dans le cadre de l'école de la communauté, sous tutelle du ministère de l'Education israélien, que les principes du Divine Life Style sont partiellement enseignés. Le dispositif éducatif à Kfar Hashalom fait lui-même partie du cadre du Divine Life Style et y est intégré sous le nom de Dedication. Des cours portant sur l'histoire

${ }^{24}$ Cette division entre jeunes générations et adultes n'est pas entièrement satisfaisante. La communauté des African Hebrew Israelites est en fait divisée en trois classes d'âge dotées chacune d'une représentation au niveau politique et de droits et de devoirs spécifiques. La Youth in the Move rassemble les jeunes de treize ans jusqu'à la majorité à vingt ans révolus. Les Chosen First Fruits rassemble les jeunes adultes jusqu'à quarante ans et la Sacred Sainthood, les adultes qui ont dépassé quarante ans. Nous appellerons ici « jeunes générations » les membres de la Youth in the Move qui sont, contrairement à leurs parents, majoritairement nés en Israël. 
de la communauté, sur le Divine Life Style et sur la lecture de la Bible sont dispensés par des instituteurs de Kfar Hahsalom.

Parmi ce corpus de matières, la musique et la pratique musicale sont abordées dans leur rapport à la liturgie, mais aussi dans une perspective morale s'appuyant sur les préceptes de Ben Ammi détaillés plus hauts. La musique y est enseignée d'un point de vue théorique, comme un élément du Divine Diet, c'est-à-dire ce qui dans le Divine Life Style est relatif à l'alimentation. Celle-ci se doit de remplir à la fois une fonction biologique et spirituelle: elle répond à un certain type de besoin mais elle comporte potentiellement un risque d'empoisonnement. L'enseignement théorique se concentre donc avant tout sur un exercice de discernement moral entre la « bonne » et la « mauvaise » musique.

Cette dimension théorique d'apprentissage du fait musical se double d'un aspect plus pratique portant sur la transmission des normes qui structurent la figure du musicien-prophète. Dans le cadre d'activités musicales scolaires et extrascolaires, les enfants sont globalement encouragés à développer leurs talents musicaux, soit sous la tutelle d'un enseignant pour les chorales scolaires, soit d'un musicien lors de cours particuliers, parfois les deux.

Au sein de l'école de la communauté, l'aspect pratique de l'enseignement musical prend la forme de chorales dont les chants, se présentant sous la forme d'hymnes, mobilisent les symboles nationaux du Kingdom of Yah comme le terme Yah Khai. Ce dernier est la devise nationale du Kingdom of Yah. Il signifie «Yah vie » ou «Dieu vie» et rappelle en substance que le Dieu d'Israël est un Dieu vivant, et que les African Hebrew Israelites appliquent au quotidien les lois qu'il édicte sur l'existence terrestre.

Ces chorales se produisent le plus souvent dans un cadre cérémoniel à destination de la communauté mais aussi, et cela est assez récent, lors d'échanges culturels avec des familles que les African Hebrew Israelites qualifient d' " afro-palestiniennes 25 ». C'est le cas des Young Lions, chorale de garçons âgés de dix ans en moyenne, encadrés par une enseignante et chanteuse de gospel surnommée la Mighty Lionness dans la communauté. À Jéricho, face à un public palestinien, mais aussi lors de la cérémonie de Shabbat à Dimona, ils chantent et dansent en appuyant les paroles par des expressions sérieuses et déterminées, leur hymne le plus connu, Shinning :

«You gave me your light and I gonna shine it! Yah Khai !

You gave me your love and I gonna claim it! Yah Khai !

${ }^{25}$ Les African Hebrew Israelites s'efforcent de construire un « dialogue » sous forme d'échanges culturels (concerts, repas...) avec des familles palestiniennes noires. Cet effort se couple à une revendication centrale de la part du leadership communautaire, celle de la "présence Africaine ancienne en Terre sainte». Les African Hebrew Israelites se proposent de fédérer toutes les populations noires vivant en Israël, après avoir prouvé leur origine israélite, en présentant le Divine Life Style et le gouvernement de Ben Ammi comme des alternatives au conflit israélo-palestinien. 
You gave me your love and I gonna live it! Yah Khai !

You gave me your love and I gonna spread it! Yah Khai !

Shinning for $\mathrm{abba}^{26}$, shinning for my ima,

Shinning for my saba, shinning for savta,

Shinning for family, shinning for my leaders,

Shinning for my kingdom, shinning for my Yah »,

«Shinning », Les Young Lions, 2012, retranscription personnelle.

On le voit bien ici, l'aspect pratique de l'enseignement de la musique à Kfar Hashalom est à double tranchant. Une chorale comme les Young Lions est bien pensée comme une plateforme pédagogique qui permet tout d'abord de transmettre aux enfants des savoir-faire propres aux techniques de chants telles qu'elles sont pensées dans la communauté par le biais de la figure du musicien-prophète. Grâce à ces chants et à la discipline de groupe, les enfants sont invités à développer une approche du fait musical conforme au cadre du Divine Life Style qu'ils peuvent s'ils le souhaitent approfondir dans leur parcours individuel.

Mais cette chorale est également pensée comme un outil de communication communautaire, une plateforme de diffusion du discours nationaliste des African Hebrew Israelites et de la doctrine de Ben Ammi Ben Israël. C'est donc également un apprentissage "politique » qui est proposé aux Young Lions, en leur permettant d'endosser le rôle de représentants de la communauté et de jeunes ambassadeurs, au même titre que les Soul Messengers avant eux, érigés en toile de fond comme modèles de réussite.

Les Young Lions ne sont toutefois pas les seuls témoins de l'importance accordée aux pratiques musicales dans le cadre éducatif de Kfar Hashalom. La visibilité de certains membres de la communauté comme Eddy Butler, représentant Israël à l'émission de télévision « Eurovision » en 2006, les succès des Soul Messengers collaborant régulièrement avec les chaînes de télévision israéliennes ou les collaborations du New World Fire Choir avec des artistes comme Stevie Wonder, ont convaincu de nombreux jeunes de Kfar Hashalom que la pratique musicale leur ouvrirait des portes sur un débouché professionnel. Ainsi, de nombreux jeunes se lancent dans des expériences musicales, profitant des fréquentes cérémonies et conférences de la communauté, pour se produire en public et perfectionner leur répertoire. Les pratiques musicales à vocation éducative et cérémonielle, s'inscrivent largement dans le cadre orthodoxe des Songs of Delivrance. Celui-ci, loin d'être perçu comme une contrainte, est plutôt considéré comme un modèle de production musicale "familial», qui a fait ses preuves dans le passé et qui dispose de réels moyens de diffusion, par le biais du studio de Dimona, et dont les membres de la communauté seront, les premiers bénéficiaires.

${ }^{26}$ En hébreu, abba, ima, saba et savta signifient respectivement père, mère, grand-père et grandmère. 
La musique produite dans un cadre communautaire, articulée à une logique éducative envers la jeunesse joue un rôle important à Kfar Hashalom par son effet fédérateur et normatif. Pourtant, elle n'empêche pas cette jeunesse, entourée et partiellement intégrée à la société israélienne, disposant d'un accès aux nouvelles technologies de l'information et de la communication, de partir à la rencontre de nouveaux horizons musicaux, sur le plan de la pratique comme sur le plan de l'écoute. Ainsi, la dissémination des styles musicaux comme le rap et gangsta rap ou les catégories tant décriées par Ben Ammi comme la variété ou la world music au sein de la jeunesse du Kingdom of Yah est un phénomène qui n'a pas échappé à la communauté dans son ensemble. Interroger ce phénomène conduit à dessiner une ligne de démarcation plus ou moins saillante entre les différentes générations de Kfar Hashalom, qui développent des stratégies de négociation internes inédites dans l'histoire de la communauté, renforçant l'idée des pratiques musicales comme un enjeu identitaire transgénérationnel. Nous prendrons comme exemple l'émergence de la musique rap au sein de la communauté.

\section{LA MUSIQUE COMME ENJEU IDENTITAIRE TRANSGÉNÉRATIONNEL : L'ÉMERGENCE DU RAP À KFAR HASHALOM}

\section{Le cadre culturel du Divine Life Style à l'épreuve de la mutation culturelle}

L'évolution des pratiques musicales chez les African Hebrew Israelites, montre que l'idéologie communautaire mise en place par Ben Ammi Ben Israël s'apparente tout à fait à un cadre conceptuel prévu pour délimiter des pratiques culturelles, et, dans le domaine musical, délimiter ce qui peut être considéré comme de la musique et ce qui ne peut pas l'être. Ce cadre se double d'un dispositif de sanction des bonnes pratiques musicales passant par du label Songs of Delivrance et du studio d'enregistrement de Kfar Hahsalom.

Ce cadre culturel s'est enrichi au fil des années de nombreux éléments discursifs et de nouvelles pratiques, interprétées, notamment par Martina Könighofer (1998: 84) comme de "nouvelles traditions» que les African Hebrew Israelites ne considèrent que "redécouvrir » au fur et à mesure de leur ascension divine au sein du Kingdom of Yah.

Sans rentrer dans le détail des modalités de validation du savoir à Kfar Hashalom, présentons-en simplement quelques aspects pour nous permettre de comprendre le processus de circulation et d'intégration d'une idée, qui pourra devenir, éventuellement, une nouvelle pratique culturelle intégrée au Divine Life Style.

Les African Hebrew Israelites considèrent eux-mêmes leur culture comme «dynamique», en perpétuelle évolution, qu'ils qualifient $\mathrm{d}^{\prime}$ "ascension spirituelle ». Un des adages favoris de Ben Ammi, fréquemment rappelé tant 
lors des conférences de la School of the Prophets ${ }^{27}$ que lors des services religieux est: «Tout commence par une pensée» 28 . Cette idée même de "culture dynamique » est au fondement de la politique de développement culturel de Kfar Hashalom: si l'on attend des érudits, des ministres ou des prêtres de générer de nouvelles idées, n'importe qui au sein de la communauté est susceptible de proposer un nouveau concept qu'il souhaiterait porter à la connaissance du leadership communautaire.

Cette catégorie d'idée ou de découverte est assimilée à celle de "révélation ». Le savoir est pensé comme une chaîne de révélations divines qui s'articulent entre elles pour constituer un maillage conceptuel divin et cohérent, trouvant son expression pratique dans le Divine Life Style.

Ainsi, les ministères de l'Information, de la Communication et de l'Education sont perpétuellement en train de chercher (dans la littérature biblique ou profane, sur Internet ou par des échanges avec d'autres groupes ou institutions nationales ou internationales) de nouvelles idées ou pratiques pour enrichir ce savoir communautaire. Qu'ils puisent leur inspiration dans la Bible ou dans des groupes extérieurs à la communauté, chaque nouvel élément sera confronté avec le texte biblique et le modèle du Divine Life Style.

Généralement, les idées émises par le leadership ou par Ben Ammi sont discutées au Conseil des princes et des ministres et décrétées comme officiellement applicables par les prêtres lors des services religieux en tant que nouvelles règles ou ajustements du Divine Life Style. En revanche, les initiatives et nouvelles pratiques émanant des autres habitants de Kfar Hashalom suivent généralement le chemin inverse, en passant d'abord par une consultation des prêtres, pour être portées à l'attention des ministres puis de Ben Ammi afin d'être décrétées ou non comme applicables. Le cadre de validation d'une nouvelle pratique ou tradition obéit à des logiques que nous ne sommes pas en mesure de détailler dans le présent article mais dont nous nous contenterons de dire qu'il se base, encore une fois, sur le respect des règles du Divine Life Style et sur son possible rattachement au texte biblique sachant que des facteurs politiques et économiques conditionnent également tout développement à Kfar Hashalom. C'est un cadre contraignant et totalitaire par lequel est censée passer toute innovation sociale, culturelle ou technique.

Le cas de l'évolution des pratiques musicales dans la jeunesse de Kfar Hashalom, et particulièrement l'émergence de la musique rap n'a pourtant suivi aucun de ces schémas. Le changement est intervenu progressivement, corrélativement à l'intégration des nouvelles technologies de l'information et de la communication, au développement des technologies informatiques portatives et à la standardisation des formats audio numériques à destination du grand public (MP3).

27 Centre de formation du leadership communautaire.

28 « Everything starts with a thought». 
Aujourd'hui, avec pour principal support technique le couple ordinateur et téléphone portable à carte mémoire, appareils photo ou vidéo intégrés, éventuellement doté d'un accès à Internet, les jeunes de la communauté se sont très vite appropriés de nouvelles modalités de production et de diffusion musicale, parallèles à celles de Kfar Hashalom mais ne visant pas nécessairement les mêmes objectifs.

La plupart des adultes posent un regard ambivalent à la fois sur les conditions de circulation de ces nouvelles pratiques ou nouvelles musiques dans la communauté, et sur la charge symbolique que ces dernières comportent pour la jeunesse.

\section{La musique rap à Kfar Hashalom : contexte d'émergence et divergence des regards}

A partir des années 1990, nous l'avons vu, l'amélioration des conditions de vie à Kfar Hashalom a amené à un certain changement dans la façon de vivre des African Hebrew Israelites. Selon plusieurs porte-paroles de la communauté (tous adultes) et plusieurs commerçants de Dimona, ce changement est majoritairement interprété par un accès plus aisé à des ressources financières et une augmentation du confort de vie individuel et collectif.

Ces mutations se sont accompagnées, selon certains membres du leadership qui le déplorent, de l'essor d'un "certain individualisme». C'est à une tendance à l'individualisme et au «laxisme» des parents que nombre d'adultes de la communauté imputent la dissémination de "nouvelles images », de "nouveaux sons » et «influences néfastes » parmi la jeunesse de la communauté, avec pour corollaire direct la généralisation des nouvelles technologies de l'information chez les jeunes générations de Kfar Hashalom.

Le premier regard jeté sur des pratiques musicales comme le rap est donc d'abord un regard désapprobateur qui se fond dans une critique plus globale de l'évolution de la communauté dans son ensemble. En témoignent les nombreuses publications de Ben Ammi Ben Israël depuis les années 1990 (d'ailleurs largement diffusées sur Internet) et le nombre croissant de conférences organisées par la School of the Prophets à propos des dangers de la musique «du diable» sur les enfants ${ }^{29}$. Dans le cas de la musique rap, la critique se base sur trois éléments principaux.

D'une part, le rap, originaire des Etats-Unis et particulièrement des grandes agglomérations du Nord-Est, est perçue dans la communauté dès ses premiers développements dans les années 1970, comme un style de musique avant tout lié au contexte de vie des Noirs au sein de la "grande Babylone euro-

\footnotetext{
${ }^{29}$ En 2010, nous avons assisté au Cycle annuel de cours et de conférences sur des sujets divers touchant à la communauté. Il s'agissait du National History Month intitulé : " A Decade for the Children ». Le thème central y était l'enfance et une des conférences les plus importante était intitulée "Who in the Hell put the Devil in our Music? ("Qui diable a mis le démon dans notre musique?»).
} 
américaine ». D'un point de vue musical, ni les rythmes syncopés du rap ni sa forte inspiration de la musique funk ne rentrent dans le cadre musical des Songs of Delivrance. Si Ben Ammi rattache la «musique noire» aux Musiques du Temple en procédant, on l'a vu à un inventaire généalogique de catégories musicales comme le gospel, la soul ou le blues, il ne fait pas rentrer une catégorie "rap» dans le panthéon des musiques divines. Les adultes se trouvent donc confrontés à un style musical qui, s'il ne leur est pas inconnu, ne leur parle pas musicalement de la même façon qu'aux jeunes générations ; d'autant qu'il prendra une ampleur mondiale à partir des années 1980 et demeurera, jusqu'à aujourd'hui, un des marchés musicaux les plus importants au monde.

D'autre part, le rap et particulièrement la catégorie de gangsta rap, est accusée de véhiculer des images et des symboles non seulement incompatibles avec le Divine Life Style mais également franchement antithétiques. La célébration de l'argent, le culte de la réussite matérielle et l'exhibition des armes à feu (considérés par les African Hebrew Israelites comme des symboles du combat fratricide entre Noirs) scandés au moyen d'un langage injurieux, sont les principaux éléments retenus par les leaders et les adultes de la communauté. L'écoute par les jeunes de cette musique est donc globalement désapprouvée depuis des années, et elle est rapidement associée à l'idée de «filer un mauvais coton».

Enfin, pour de nombreux jeunes, le rap est, de manière tout à fait admise, une sorte de lucarne ouverte sur les Etats-Unis d'Amérique qu'ils ne connaissent, pour la plupart, que par les récits de leurs parents, des liens familiaux plus ou moins proches et les «invités » de la communauté qui viennent majoritairement des Etats-Unis.

La perception que les jeunes African Hebrew Israelites ont des Etats-Unis d'Amérique ne semble complètement s'accommoder ni de la vision qu'en ont leurs parents, qui y voient avant tout un espace maléfique, ni de celle relayée par les images de la culture hip-hop. Pourtant, les Etats-Unis fascinent et des rappeurs afro-américains comme Akon, Jay- $Z$ ou Jason Derulo offrent à la jeunesse de Kfar Hashalom des référents musicaux rattachés à une musique qui "leur parle", mais qui sont aussi des références partageables avec le monde entier.

Les adultes, eux, perçoivent cette tendance de "reconnexion» avec les Etats-Unis de différentes façons. Pour certains, elle mènerait à terme à un véritable scénario catastrophe de désertion de Kfar Hashalom par ses membres les plus jeunes. Pour d'autres, moins alarmistes, cet intérêt envers le rap et la culture hip-hop est un processus logique, lié au contexte d'implantation bien particulier de la communauté en Israël. Certains évoquent un déracinement que vivent au quotidien les jeunes African Hebrew Israelites, un déracinement pourtant nécessaire dans le cadre du projet rédempteur de la communauté mais qui n'exclut pas une connaissance des Etats-Unis dans le cadre du travail communautaire ou des affaires. 
Interrogés sur ces considérations, certains jeunes répondent le plus souvent avec désinvolture : "C'est juste de la musique! ». Mais cet argument vite repris est critiqué par un ministre du Peuple : "Ce n'est pas juste de la musique, on ne peut pas dire ça (...) Toutes ces images négatives pénètrent leurs esprits et les influencent à agir comme des imbéciles (...) Ce qu'ils ont besoin de comprendre c'est ça, car s'ils ne sont pas capables de le comprendre, ils ne seront jamais non plus capables de comprendre la vérité et ils ne feront que reproduire les idées qui nous ont fait partir d'Amérique (...) Tu peux vivre vingt ans dans le Kingdom, porter une kippa, aller au service de Shabbat, mais si tu n'es pas capable de comprendre et d'appliquer la vérité dans ta propre vie, tu n'en feras jamais partie » (entretien avec Sar Elyakim, Dimona, le 15 mars 2010).

Ce ministre du Peuple exprime ici un point de vue caractéristique du leadership communautaire, très proche en substance de la pensée de Ben Ammi Ben Israël. D'autres adultes et parents ont cependant une approche critique plus contrastée. Uvadiah, mère de quatre jeunes filles explique son point de vue : "On ne peut pas empêcher les enfants de sortir pour toujours (...) Je n'aime pas que les filles écoutent cette musique et elles le savent, mais pour moi l'important est qu'elles comprennent la limite (...) Je sais qu'elles savent où est la limite car elles peuvent discerner ce qui est bon pour elles » (entretien avec Uvadiah, Dimona, le 12 Avril 2010).

Dans ces deux exemples pourtant, $c^{\prime}$ est l'affichage d'un rejet pour le rap qui domine. Cette critique "par le haut» trace les contours d'une modalité d'écoute ou d'une pratique de la musique rap privilégiant le secret: une pratique désapprouvée et légèrement honteuse.

Pourtant, les marqueurs identitaires en lien avec le monde du rap se multiplient chez les jeunes de Kfar Hashalom. À l'école, au collège sur les terrains de basket-ball du gymnase s'affichent pantalons baggy, chaussures de basket, casquettes de base-ball, personnalisations d'uniformes des plus variées ou encore l'exhibition de moins en moins dissimulée de chaînes et bagues massives, caractéristiques de la culture gangsta rap. Ce que ces jeunes perçoivent comme les éléments de la culture hip-hop américaine deviennent des référents identitaires importants, alimentant du même coup les craintes des adultes d'une « chute spirituelle vers les Etats-Unis ».

Enfin, ce regard critique se double d'une réflexion plus large sur le devenir de la communauté en Israël, et particulièrement sur la façon dont les enfants, considérés dans l'idéologie de Ben Ammi comme les "graines sacrées" revenues à l'état d'innocence et préservées du mal, négocient leur place dans ce pays.

L'histoire communautaire fait, nous l'avons vu, la part belle au parcours de Ben Ammi et des "pionniers » du Libéria et d'Israël en soulignant les nombreux «sacrifices » qui ont été faits pour offrir à leurs enfants un monde meilleur. Toutefois, malgré les améliorations des relations entre Israël et la communauté, la plupart des jeunes n'ont toujours pas accès à la citoyenneté 
israélienne malgré leur participation au Service national. Nombres d'entre eux soulignent également les difficultés à trouver un travail en Israël, et les inscriptions dans les universités israéliennes sont rarissimes pour des raisons financières et administratives. Une partie de la population israélienne vivant à Dimona signale également depuis plusieurs années l'émergence d'une délinquance (très minoritaire) de certains jeunes African Hebrew Israelites basée sur une logique de « gang ».

Ainsi, si certains fidèles de Ben Ammi considèrent que les enfants de la communauté sont « bénis » et " préservés » d'un monde extérieur néfaste par le cadre de vie à Kfar Hashalom, d'autres pensent que ces enfants ont, et continuent de largement contribuer à l'ensemble des « sacrifices » auxquels les African Hebrew Israelites ont dû procéder. Face à un phénomène comme l'émergence du rap, et, apparemment, des indissociables éléments langagiers, comportementaux et vestimentaires qui l'accompagnent, la communauté a engagé une réaction inédite dans son histoire en procédant à un aménagement $\mathrm{du}$ cadre culturel du Divine Life Style pour y accueillir, selon certaines conditions, ses jeunes rappeurs et amateurs de rap.

Kfar Hashalom célèbre en effet depuis quelques années la création de ses premiers groupes de rap. Comment ce phénomène a-t-il vu le jour et quelles sont ses implications? Nous tenterons de dégager quelques pistes de réflexion en présentant un de ces groupes, The Unknown et la façon dont il perçoit son activité dans le cadre actuel de la communauté.

\section{L'émergence du rap African Hebrew Israelites, le cas du groupe The Unknown}

Dans l'état de nos connaissances, il nous est impossible de dater la création du groupe de The Unknown. Sur la base de nos observations et échanges avec la communauté, il est toutefois possible de dégager deux éléments qui viennent alimenter notre réflexion sur les pratiques musicales comme marqueur identitaire transgénérationnel à Kfar Hashalom.

Tout d'abord, sans donner de dates précises, l'apparition du rap dans la communauté précède de plusieurs années son intégration dans le cadre de ses activités musicales. On peut, sans prendre de risques, qualifier cette intégration de « récente »: dans les brochures de présentation des activités musicales de la communauté destinées à la clientèle nationale ou internationale, il n'est fait mention nulle part des groupes de rap communautaire dans la dernière édition qui remonte à 2009. D'autre part, en dépit de plusieurs apparitions sporadiques sur scène lors de différentes cérémonies à Dimona (jour d'Indépendance en 2010, National History Month en 2010), les groupes de rap de Kfar Hashalom demeurent pour nombre d'adultes, musiciens ou non, de parfaits « inconnus ».

Deuxièmement, et la teneur du discours du jeune rappeur Elazar Ben Israël sera là pour en témoigner, l'intégration de cette forme musicale à Kfar Hashalom n'apparaît ni comme étant le résultat d'un triomphe de la jeunesse 
brandissant les symboles du hip-hop à la barbe du leadership communautaire, ni comme une opération de "calibrage" parfait de ce dernier envers une modalité de pratique musicale jugée dérangeante. Cette intégration apparaît plus comme le fruit d'une longue, complexe négociation, parfois conflictuelle, tout d'abord entre les jeunes générations de la communauté et leur contexte de vie israélien, puis entre eux-mêmes et leurs parents, réfléchissant selon des modalités et des priorités différentes à leur futur commun.

The Unknown est présenté dans un bref reportage disponible sur Youtube et produit par la chaîne numérique Current en 2010. Ce collectif basé à Tel-Aviv, est dirigé par deux jeunes rappeurs, Elazar Ben Israël ${ }^{30}$ et Avi Shakar Ben Israël. Au cours de ce bref documentaire, des journalistes suivent les membres du groupe qui vont rendre visite à leur famille à Dimona. C'est l'occasion pour eux de présenter la communauté et son histoire, ainsi que de donner leur point de vue en tant que jeune rappeur African Hebrew Israelite en Israël.

Elazar Ben Israël présente The Unknown comme un groupe « authentique », originaire de la "Terre promise », n'ayant aucun rapport avec les EtatsUnis et bien implanté en Israël. Toutefois, lors d'une interview, le groupe annonce qu'il s'envole justement pour une tournée aux Etats-Unis :

«Je ne suis jamais allé aux Etats-Unis, je ne suis jamais parti du pays, je ne suis jamais parti nulle part, vraiment. Israël est tout ce que je connais pour l'instant (...) Vous savez, je suis né dans la communauté, pas de violence, pas de tabac, pas d'alcool, pas de sexe avant le mariage, pas de viande : nous sommes végétaliens, pas d'injures, nous sommes totalement en sûreté je veux dire (...). Ils [les parents] nous ont déconnectés du monde, en nous mettant dans une coquille et en nous protégeant (...). Maintenant que nous sommes libérés de cette coquille, on avance libre et tout ça mais on se rappelle encore de tout ce qu'on nous a appris (...). Et maintenant, quarante ans plus tard regardez moi ! Je veux dire, c'est la fierté que nous avons, des droits, nous avons la citoyenneté, nous avons le droit de rejoindre l'armée, nous avons tout (...).

Vous savez, notre plus grand rêve n'est pas d'atteindre les "Etats-Unis d'Amérique", les grandes villes et les rêves, vous savez. Je veux dire, on revient en Amérique pour prouver notre point de vue (...). Comme des hommes libres, une fois encore, on va revenir là-bas, on va faire trembler la scène, vibrer le micro ! Une prise de contrôle totale, voilà ce que ça va être ! (...)

Le peuple Noir ne le saura jamais, il ne le saura jamais jusqu'à ce qu'on lui dise ${ }^{31}$ !» (Elazar Ben Israël, 2010 ; traduction personnelle ${ }^{32}$ ).

30 Les attributions patronymiques à Kfar Hashalom obéissent à des modalités complexes que nous ne détaillerons pas ici. Nous nous contenterons de signaler le fait que le patronyme Ben Israël ne signifie pas nécessairement une relation de filiation avec le leader Ben Ammi Ben Israël.

${ }^{31}$ Cette dernière phrase fait référence à l'idée des African Hebrew Israelites selon laquelle les descendants de ceux qui ont été emmenés en esclavage depuis l'Afrique de l'Ouest jusqu'en Amérique sont les vrais hébreux de la Bible.

32 «I never been to the States, never left the country, I never been anywhere really. Israel is all I know in the meantime (...) You know (...) I was born in the community, no violence, no 
Dans cette brève retranscription du discours du jeune Elazar Ben Israël, on voit poindre une tension entre deux pôles distincts : d'où il vient et où il va.

Le premier élément mis en avant est l'endroit dont il vient, le lieu qu'il connaît et qu'il estime être "chez lui ». De manière assez nette, il délimite l'espace israélien comme son pays, le seul où il soit allé précise-t-il, il y inclut la communauté, qu'il perçoit comme un espace intégré dans le premier.

Elazar décrira l'expérience de la vie à Kfar Hashalom comme une "déconnexion" du monde, que ses parents auraient opéré à des fins de protection, de préservation de maux identifiés et énumérés de manière très explicite (violence, tabac, alcool...). La «coquille» à laquelle il fait référence représente Kfar Hashalom et son univers de sens, marqué, en ce qui concerne la jeunesse par la symbolique des "graines sacrées » à préserver de l'influence du monde extérieur. Elazar estime cependant qu'il est, ainsi que les jeunes de sa génération, désormais libéré de cette "coquille»: il a atteint sa majorité, effectué ses années de volontariat au sein de Tsahal et «avance libre » fort de tout ce qu'on lui a appris. Ce faisant, il mobilise la grille de lecture communautaire concernant l'enfance et la jeunesse en la brandissant comme un signe identitaire, une marque de garantie qui justifie le nouveau cap qu'il souhaite prendre. Il s'empare à bras le corps de l'idéologie de Ben Ammi en aménageant le rôle que ce dernier a conçu pour la jeunesse : celui des " graines sacrées » appelées à régner sur Terre et être les reflets mêmes de l'idée de vérité structurant le Divine Life Style.

Pourtant, ce n'est pas vers Israël et la vie à Kfar Hashalom que se tourne ce jeune rappeur.

Le deuxième pôle qui sous-tend son discours semble se structurer de manière assez claire autour des Etats-Unis d'Amérique, dont les images évoquées, si grandioses soient-elles, se résument finalement à « des grandes villes et des rêves ».

Or, ce n'est pas cela que part chercher le groupe en s'envolant vers les scènes états-uniennes. Le groupe y va pour "prouver son point de vue ", " prendre le contrôle » et dire au peuple noir ce qu'il a besoin d'entendre : il «est» le peuple hébreu, il doit prendre conscience de ses origines et

smocking, no drinking, no sex before marriage, no eating meat: we are vegetarian, no cursing, we keep totally safe I mean...(...) they [leurs parents, immigrés en Israël] disconnected us from the world, putting us in a shell and protecting us (...) now that we are free from that shell we're running free and everything but we still remember everything we were told (...) and now, forty years later look at me ! I mean this is the proud that we have, rights, we have citizenship, we have the right to join the army, we have everything (...) You know our biggest dream is not to make it to the "United States of America ", big cities and dreams you know, I mean we're going back to America to prove our point I mean, (...) as free men, once again, come back over there we gonna rock the stage, rock the mic! Global takeover that's what it's gonna be (...) Black people will never know, they'll never know until we tell'em » Elazar Ben Israël, 2010. 
embrasser la voie de la rédemption. Ce deuxième pôle se nourrit donc largement du premier puisque c'est, selon les éléments de discours d'Elazar, un rôle du musicien-prophète, justifié par un statut de "graine sacrée » qui génère l'impulsion du départ, qui délimite une destination et un objectif : en un mot, une " mission ».

Elazar se propose de déployer sa pratique musicale à un double niveau qui correspond tout à fait à ce que Ben Ammi explique que l'on doit attendre de quelqu'un qui se définit comme musicien : en tant que rappeur et en tant que prophète.

Si l'exemple du collectif The Unknown n'illustre qu'un cas particulier de l'intégration du rap à Kfar Hashalom, plusieurs éléments intéressants émergent du discours d'Elazar, et témoignent d'une «négociation » du cadre culturel du Divine Life Style dans la communauté.

Tout d'abord, nous l'avons vu, l'édifice politique de Kfar Hashalom est largement dominé par une population adulte, qui définit dans une mesure écrasante le cadre normatif, sur le plan social et culturel au sein de la communauté. Pourtant, l'émergence du rap à Kfar Hashalom chez les plus jeunes a eu pour résultat une intégration (balbutiante) de cette musique comme un nouveau support de déploiement du message communautaire. C'est d'ailleurs apparemment la seule condition de déploiement d'une «tendance rap » dans les musiques du Kingdom of Yah. Les rappeurs de The Unknown affichent clairement un profond engagement envers les idées de Ben Ammi Israël, mais ils utilisent dans le même temps ce discours comme un levier dans le cadre d'une mise en scène de soi, individuelle et collective, particulièrement dans le cadre d'une vidéo promotionnelle comme celle de la chaîne Current. Dans le cas de The Unknown, on assiste bien à un aménagement de la catégorie de "graine sacrée », pensée par les adultes et jouée par leurs enfants, dans le déploiement du rap communautaire.

Là où les artistes de gangsta rap mobilisent le caractère violent et dangereux de leur contexte de vie comme un symbole fort à destination des autres rappeurs, $c^{\prime}$ est dans une symbolique inverse, bien que reposant sur les mêmes ressorts, qu'Elazar va mobiliser son statut de "graine sacrée »: il vient du royaume de Dieu, le seul havre de paix et de liberté pour le peuple noir en ce monde et il vient pour délivrer un message.

Le rap communautaire de The Unknown propose d'inscrire l'idéologie communautaire au sein d'un espace musical alternatif incluant de nouvelles modalités de pratique et de circulation de la musique, ainsi que la construction de nouveaux réseaux transnationaux.

\section{CONCLUSION}

Finalement, au travers de l'intégration de nouvelles modalités de pratiques musicales à Kfar Hashalom, si peu revendiquées soient-elles par le leadership 
communautaire, les African Hebrew Israelites font peut-être face pour la première fois de leur histoire à un phénomène de mutation culturelle et sociale de grande ampleur mettant en jeu le cadre politique et culturel édifié par Ben Ammi Ben Israël. Le fil rouge de l'évolution des pratiques musicales à Kfar Hashalom a également révélé une vraie complexité dans les rapports intergénérationnels par la mise en discussion du cadre musical des Songs of Delivrance et plus largement, du cadre du Divine Life Style initié par Ben Ammi Ben Israël.

Cette mise en discussion du cadre du Divine Life Style, au caractère parfois conflictuel, se cristallise autour de pratiques et de symboles émergeants de la classe d'âge la plus jeune, délimitant un nouveau cadre culturel, transnational, où le rap et ses jeunes représentants ont désormais toute leur place lorsqu'ils articulent leur pratique musicale, et c'est là leur unique créneau, avec le rôle du musicien-prophète du Kingdom of Yah.

L'exemple du collectif The Unknown, ne doit pourtant pas occulter le caractère extrêmement minoritaire du phénomène du rap communautaire, pas plus qu'il ne doit faire comprendre le rap comme un révélateur trop évident des lignes de démarcation intergénérationnelle dans la mesure où les pratiques musicales de la jeunesse à Kfar Hashalom s'inscrivent dans une écrasante majorité dans le cadre des Songs of Delivrance.

L'importance du phénomène rap dans la communauté se mesure par le fait que le cadre politique de Ben Ammi, pour la première fois depuis l'arrivée en Israël est remis en cause par l'émergence de pratiques culturelles qu'il ne parvient pas à complètement contrôler sur son « propre territoire » et qui vont provoquer chez lui une réaction inédite. Cette réaction de négociation du cadre culturel communautaire de la part du leadership communautaire envers la jeunesse est le produit de multiples facteurs (sociaux, économiques, politiques) qui dépassent le cadre de la pratique musicale mais qui s'expriment au travers d'elle comme un catalyseur des espoirs, des craintes et des perceptions du futur de la communauté en Israël.

\section{BIBLIOGRAPHIE}

Taahmenyah BATH SHALEAK et Ahmadiel BEN YEHUDA, Holy Garnments For Glory and Beauty, The Divine Messianic Dress Code, Dimona, Communicators Press, date d'édition non disponible.

Hans A. BAER et Merrill SINGER, African American Religion in the Twentieth Century: varieties of protest and accomodation, University of Tennessee Press, 1992.

Ben Ammi BEN ISRAEL, God the Black Man and Truth, Washington, Communicators Press, Inc, 1982.

Yvonne CHIREAU et Nathaniel DEUTSCH, Black Zion African American Religious Encounters with Judaism, New York, Oxford University Press, Inc, 2000. 
François-Xavier FAUVELLE-AYMAR, Jean-Pierre CHRETIEN et Claude-Hélène PERROT, Afrocentrismes. L'histoire des Africains entre Egypte et Amérique, Paris, Karthala, 2010.

Pauline GUEDJ, "Des "Afro-Asiatiques" et des "Africains". Islam et Afrocentrisme aux Etats-Unis », Cahiers d'études Africaines, n¹72, 2003/4, pp.739-760

Alexander Paul HARE, The Hebrew Israelites Community, University Press of America, 1998.

John L. Jr. JACKSON, "All Yah's Children : Emigrationism, Afrocentrism and the Place of Israel in Africa", Civilisations, 58/1, 2009, pp. 93-112.

Isabelle JONVEAUX, Le Monastère au travail : le Royaume de Dieu au défi de l'économie, Paris, Bayard, 2011.

Martina KÖNIGHOFER, The New Ship of Zion, Dynamic Diaspora Dimensions of the African Hebrew Israelites of Jerusalem, Wien, LIT Verlag GmbH \& Co. KG, 2008.

James E. LANDING, Black Judaism : story of an american movement, Duhram, Carolina Academic Press, 2002.

Nicole LAPIERRE, Causes Communes : des Juifs et des Noirs, Paris, Stock, 2012.

Fran MARKOWITZ, « Israel as Africa, Africa as Israel: "Divine Geography" in the personal narrative and community identity of the Black Hebrew Israelites", Anthropological Quarterly, n69, 1996, pp. 193-206.

Fran MARKOWITZ, "Soul citizenship : The Black Hebrews and the State of Israel", American Anthropologist, vol. 105, n², 2003, pp. 302-312.

Ethan MICHAELI, "Another Exodus : The Hebrew Israelites from Chicago to Dimona", in Yvonne CHIREAU et Nathaniel DEUTSCH, Black Zion African American Religious Encounters with Judaism, New York, Oxford University Press, Inc, 2000, pp. 73-87.

Notice de présentation des Songs of Delivrance, auteur non précisé, 2009, Royal Kingdom Production.

RÉSUMÉ : Dans le cadre de l'étude d'un mouvement religieux communautaire, que peut nous apporter l'approche transgénérationnelle du fait musical ? Dans cet article, nous présenterons la communauté transnationale des African Hebrew Israelites dans son centre spirituel de Dimona dans le désert du Néguev, au travers de la façon dont elle envisage la pratique musicale dans une perspective idéologique et transgénérationnelle. Nous verrons comment depuis sa fondation, la musique y est un enjeu identitaire crucial dont les modalités de pratiques sont encadrées par des règles très strictes, conditionnées par un contexte social particulier en Israël, et comment aujourd'hui, ce cadre idéologique et social est renégocié par ses jeunes générations dans le cadre de l'émergence de nouvelles pratiques musicales.

MOTS-CLÉS : African Hebrew Israelites, nationalisme noir, Israël, Etats-Unis, musique, relations transgénérationelles 